\title{
Synthesis, characterization and cytotoxic evaluation of novel derivatives of 1, 3, 4-oxadiazole containing 5-phenyl thiophene moiety.
}

\author{
VinayakAdimule $^{1,4}$, SudhaMedapa ${ }^{2}$, AdarshaHaraballiJagadeesha ${ }^{3}$, \\ LalitaSanjeev Kumar ${ }^{4}$, Prakash Kumar Rao ${ }^{1 *}$ \\ ${ }^{1}$ Mount Carmel Centre for Scientific Research and Advanced Learning, Mount Carmel College, Vasanthnagar, \\ Bengaluru-560 052, Karnataka, India \\ ${ }^{2}$ Department of Chemistry, Mount Carmel College (Autonomous), Vasanthnagar, Bengaluru- 560052 , \\ Karnataka, India. \\ ${ }^{3}$ Inorganic and Physical Chemistry, IISc, Bengaluru, Karnataka, India. \\ ${ }^{4}$ Department of Chemistry, School of Sciences, IGNOU, New-Delhi, India.
}

\begin{abstract}
In the present work a series of novel derivatives of 1,3,4-oxadiazoles containing 5-phenyl thiophene moiety has been synthesized by convergent synthetic method and studied their anticancer properties. The synthesized compounds were characterized by spectral $\left({ }^{1} H-N M R,{ }^{13} C N M R, M S\right.$ and elemental) analyses. Three cell lines were used for thecytotoxic evaluation namely, HepG2, Caco-2 and PANC-1. The synthetic chemistry involved conversion of various substituted aromatic acids into ethyl ester 2a-e. The ethyl ester was converted into corresponding carbohydrazide 3a-e. Carbohydrazides3a-e were reacted with 5-phenyl thiophene-2carboxaldehyde(5) in presence of acetic acid as catalyst and obtained novel Schiff base compounds6a-e. The Schiff base derivatives were cyclized using chloramine-T as promoter and obtained novel derivatives of 1,3,4oxadiazole 7a-e.Among the synthesised compounds, the cytotoxicity of the compound $7 \boldsymbol{b}$ i.e. 2-(4'fluorobiphenyl-3-yl)-5-(5-phenylthiophen-2-yl)-1,3,4-oxadiazole against Caco-2 cell line with $I C_{50}$ of 5.3 $\mu M$. The compound $7 \boldsymbol{e}$ i.e.2-(4-methoxyphenyl)-5-(5-phenylthiophen-2-yl)-1,3,4-oxadiazoleshowed moderate cytotoxicity against HepG2 with $I C_{50}$ of $28.4 \mu \mathrm{M}$. Rest of the compounds showed less cytotoxicity against all the three cell lines.
\end{abstract}

Key words: PANC-1, Cytotoxicity, 1,3,4-oxadiazole, 5-phenylthiophene, Anticancer

\section{Introduction}

The development of new anticancer therapeutic agents is one of the fundamental goals in medicinal chemistry. In recent years there has been a concerned search for the discovery and development of potent and selective anticancer agents. The various derivatives of 1,3,4-oxadiazoles substituted with different groups displayed wide spectrum of biological activities such as anti-inflammatory ${ }^{[1]}$, antimicrobial ${ }^{[2]}$, analgesic ${ }^{[3]}$ antituberculosis ${ }^{[4]}$, anticancer ${ }^{[5,6,7,8]}$ properties. Author envisage that by introducing 5-phenyl thiophene moiety into the 1,3,4-oxadiazole ${ }^{[9]}$ ring system(Fig. 1) would produce the synergetic effect and increases the water solubility and total polar surface area. The synthetic chemistry involved converting suitably substituted aromatic acid into corresponding ethyl ester 2a-e. Ethyl ester was further converted into carbohydrazide 3a-e. The carbohydrazides were reacted with 5-phenyl thiophene $\mathrm{e}^{[10,11]}(\mathbf{5})$ and obtained novel Schiff base ${ }^{[12,13]}$ compounds 6a-e.The Schiff base compounds were cyclized using chloramine- $\mathrm{T}^{[14]}$ and obtained novel derivatives of 1,3,4-oxadiazole 7ae(Fig. 1).The key intermediate 5-phenyl thiophene carbohydrazide(5) and 1,3,4-oxadiazole and related compounds have been described as useful building blocks for the synthesis of various heterocyclic rings. A series of 5-phenylthiophene of 1,3,4-oxadiazole ${ }^{[15]}$ derivatives7a-e(Fig. 1)have been synthesized and tested their cytotoxicity on human cancer cell lines[. Notably, the halogen substitutions were incorporated to improve the solubility of the compounds. Author envisaged that by making Schiff base compounds of various halogens substituted aromaticcarbohydrazides with 5-phenylthiophene moiety and cyclizing the Schiff base ${ }^{[16]}$ compounds using chloramine-T as promoter improves the total polar surface area and increases the water solubility. All the final compounds were characterized by spectral $\left({ }^{1} \mathrm{H}-\mathrm{NMR},{ }^{13} \mathrm{CNMR}\right.$, MS and elemental) analyses. Thus it was of interest to implement drug design and to synthesise the novel candidates by joining the 5-phenyl thiophene with different halogen substituted aromatic carbohydrazides and obtained novel derivatives of 1,3,4-oxadiazoles. 


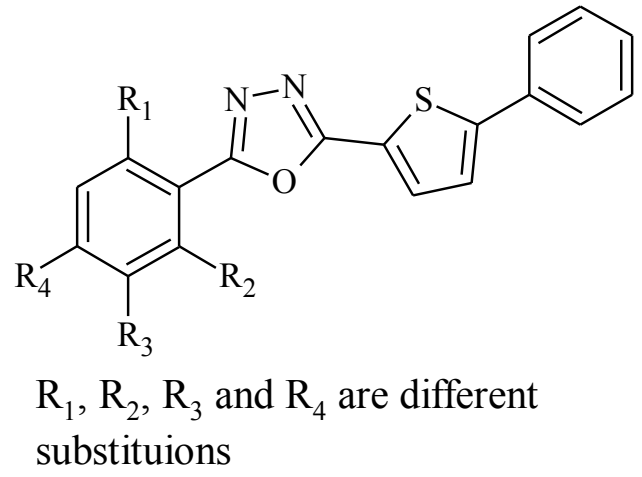

1.1 Figure 1: General structures of the 5-phenylthiophene containing 1,3,4-oxadiazole moiety

\section{Experimental:}

2.1 Materials and Methods:All the reagents, chemicals and solvents were purchased from S-d fine and Spectrochem Ltd, Bengaluru, India. ${ }^{1} \mathrm{H}-\mathrm{NMR}$ and ${ }^{13} \mathrm{C}-\mathrm{NMR}$ were recorded by Brucker $400 \mathrm{MHz}$ spectrophotometer. Melting points are determined using Buchi melting point 545. Mass spectra were recorded by Agilent 1200 series. TLC was done using F254 grade silica 60 from Merck.IR spectra was recorded by FTIR 1800 series. Whirlpool microwave (genius) was used for microwave reaction.

\subsection{Synthesis:}

\subsubsection{General procedure for synthesis of aromatic ethyl ester2a-e:}

To a mixture of acid 1a-e (1mol, lequivalent), ethyl alcohol $(50 \mathrm{~mL})$ and conc. $\mathrm{H}_{2} \mathrm{SO}_{4}$ were refluxed at $85^{\circ} \mathrm{C}$ for $6-8 \mathrm{~h}$. Progress of the reaction was monitored by TLC (thin layer chromatography), which indicated completion of the reaction. Ethyl alcohol was completely removed, residue was extracted with ethyl acetate (20 $\times 3 \mathrm{~mL}$ ), washed with brine $(10 \mathrm{~mL})$ and dried over $\mathrm{Na}_{2} \mathrm{SO}_{4}$. Ethyl acetate was concentrated under reduced pressure and obtained colourless syrup. The structure was confirmed by spectral and analytical data.

\subsubsection{General procedure for synthesis of aromatic carbohydrazide3a-e:}

To a solution of 2a-e in ethyl alcohol $(50 \mathrm{~mL})$ was added with excess of hydrazine hydrate and refluxed at $80^{\circ} \mathrm{C}$ for $10-12 \mathrm{~h}$. Progress of the reaction was monitored by TLC, indicated completion of the reaction. Reaction mixture was concentrated completely under reduced pressure, ice pieces were added and stirred. Precipitate formed was filtered and dried. The structure was confirmed by spectral and analytical data.

\subsubsection{Synthesis of 5-phenyl thiophene-2-carboxaldehyde (5):}

To a mixture of 5-bromothiophene-2-carboxaldehyde( $2 \mathrm{~g}, \quad 0.0104 \mathrm{~mol} 1$ equivalent), phenyl boronicacid $(1.405 \mathrm{~g}, 0.0115 \mathrm{~mol}), \mathrm{Na}_{2} \mathrm{CO}_{3}(2.2 \mathrm{~g}, 0.0208 \mathrm{~mol})$, tetrakis (triphenylphosphine) palladium $(0)(606 \mathrm{mg}$, $0.052 \mathrm{mmol})$ and ethyl alcohol $(100 \mathrm{~mL})$ were refluxed at $85^{\circ} \mathrm{C}$ for $10 \mathrm{~h}$.Progress of the reaction was monitored by TLC, which indicated the completion of the reaction. Ethyl alcohol was completely removed, residue was extracted with ethyl acetate $(25 \mathrm{~mL} \times 3)$, washed with brine $(10 \mathrm{~mL})$ and dried over $\mathrm{Na}_{2} \mathrm{SO}_{4}$. The crude product was purified by column-chromatography (silicagel100-200mesh), ethylacetate inhexane $(0-15 \%)$ as eluent. Yield $=$ $1.85 \mathrm{~g}$; m.p-98- $123^{\circ} \mathrm{C}$; $1 \mathrm{H}-\mathrm{NMR}\left(\mathrm{CDCl}_{3}, 400 \mathrm{MHz}\right): \delta 7.45$ (dd, $\left.2 \mathrm{H}\right), 7.52(\mathrm{~m}, 3 \mathrm{H}, \mathrm{J}=12.8 \mathrm{~Hz}), 7.62(\mathrm{~d}, 2 \mathrm{H}, \mathrm{J}=$ $6.8 \mathrm{~Hz}), 7.67(\mathrm{~d}, 1 \mathrm{H}), 8.8(\mathrm{~s}, 1 \mathrm{H})$; 13CNMR (CDCl 3 , 100MHz): 125.5, 126.5, 127, 129, 132, 136, 138, 141, 192.

\subsubsection{General procedure for synthesis of Schiff base derivatives 1,3,4-oxadiazole containing 5- phenylthiophene6a-e:}

To a mixture of carbohydrazide 3a-e, acetic acid(2-5drops) and 5-phenylthiophene-2-carboxaldehyde (5) $(1.1 \mathrm{mmole})$ were refluxed at $85^{\circ} \mathrm{C}$ for $2-3 \mathrm{~h}$. Progress of the reaction was monitored by TLC, indicated the completion of the reaction. Reaction mixture was concentrate under reduced pressure, ice cold water was added and the precipitate formed was filtered, dried. The structure was confirmed by spectral and analytical methods.

\subsubsection{General procedure for synthesis 5-phenylthiophene substituted 1,3,4-oxadiazoles 7a-e:}

To a solution of the compound 6a-e $(1 \mathrm{mmol})$ ion ethyl alcohol $(5 \mathrm{~mL})$ and Chloramine-T $(1.1 \mathrm{mmol})$ was irradiated with microwave for 60 seconds. Progress of the reaction was monitored by TLC, indicated completion of the reaction. Reaction mixture was extracted with ethyl acetate $(25 \mathrm{~mL} \times 3)$, washed with brine $(10 \mathrm{~mL})$ and dried over $\mathrm{Na}_{2} \mathrm{SO}_{4}$. The crude product was purified by column-chromatography (silicagel100200 mesh), ethylacetate inhexane $(0-75 \%)$ as eluent. 
Synthesis, characterization and cytotoxic evaluation of novel derivatives of $1,3,4-\ldots .$.

2.3 Analytical data of the compounds 7a-e:

2.3.1 2-(2,5-Dimethoxy-phenyl)-5-(5-phenyl-thiophen-2-yl)-[1,3,4]oxadiazole (7a):

Pale yellow solid; Yield 45\%; m. p 103-106 C; ${ }^{\circ} \mathrm{H}-\mathrm{NMR}\left(400 \mathrm{MHz}, \mathrm{CDCl}_{3}\right): \delta 3.85\left(\mathrm{~s}, 3 \mathrm{H}, \mathrm{OCH}_{3}\right), 3.95(\mathrm{~s}, 3 \mathrm{H}$, $\left.\mathrm{OCH}_{3}\right), 7.0195(\mathrm{~d}, 1 \mathrm{H}, \mathrm{J}=9.2, \operatorname{Ar}-\mathrm{H}), 7.082(\mathrm{dd}, 1 \mathrm{H}, \mathrm{J}=12 \mathrm{~Hz}), 7.298(\mathrm{~d}, 3 \mathrm{H}, \mathrm{J}=8 \mathrm{~Hz}), 7.374(\mathrm{t}, 1 \mathrm{H}, \mathrm{J}=$ $7.2 \mathrm{~Hz}), 7.435(\mathrm{t}, 1 \mathrm{H}, \mathrm{J}=14.8 \mathrm{~Hz}), 7.534(\mathrm{~d}, 1 \mathrm{H}, \mathrm{J}=3.2 \mathrm{~Hz}), 7.675(\mathrm{t}, 1 \mathrm{H}, \mathrm{J}=8.4 \mathrm{~Hz}), 7.799(\mathrm{q}, 3 \mathrm{H}, \mathrm{J}=14.8 \mathrm{~Hz})$; ${ }^{13} \mathrm{C}-\mathrm{NMR}\left(\mathrm{CDCl}_{3}, 100 \mathrm{MHz}\right): 21.6,56.5,77.16,113.5,114.7,119.4,124,126.35,128.8,129.53,130.8,133.2$, 139.3, 143.6, 149.3, 152.4, 153.6, 160.7, 162.7; IR (KBr, cm $\left.{ }^{-1}\right)(\mathrm{C}-\mathrm{H})$ 2935, (CH, w) 3322, (C-O) 1180, (C-F) 874, (C-S) $1151\left(\mathrm{CH}_{2}\right)-2765$, (NH) 3320; MS(ESI) m/z : $365[\mathrm{M}+\mathrm{H}]^{+}$; anal. Calculated for $\mathrm{C}_{20} \mathrm{H}_{16} \mathrm{~N}_{2} \mathrm{O}_{3} \mathrm{~S} ; \mathrm{C}_{\text {, }}$ 65.92; H, 4.43; N, 7.69; O, 13.17; S, 8.80; found C, 65.96; H, 4.47; N, 7.70; O, 13.18; S, 8.82.

\subsubsection{2-(4'-Fluoro-biphenyl-3-yl)-5-(5-phenyl-thiophen-2-yl)-[1,3,4]oxadiazole(7b):}

Off white solid; Yield 76\%; m. p $112-116^{0} \mathrm{C} ;{ }^{1} \mathrm{H}-\mathrm{NMR}\left(400 \mathrm{MHz}, \mathrm{CDCl}_{3}\right): \delta 7.374(\mathrm{q}, 2 \mathrm{H}, \mathrm{J}=15.2 \mathrm{~Hz}), 7.439$ $(\mathrm{q}, 3 \mathrm{H}, \mathrm{J}=16.8 \mathrm{~Hz}), 7.502(\mathrm{t}, 2 \mathrm{H}, \mathrm{J}=14.8, \mathrm{Ar}-\mathrm{H}), 7.610(\mathrm{t}, 1 \mathrm{H}, \mathrm{J}=15.6 \mathrm{~Hz}), 7.676(\mathrm{~d}, 4 \mathrm{H}, \mathrm{J}=7.6 \mathrm{~Hz}), 7.78(\mathrm{~d}$, $1 \mathrm{H}, \mathrm{J}=8 \mathrm{~Hz}), 7.822(\mathrm{~d}, 1 \mathrm{H}, \mathrm{J}=4 \mathrm{~Hz}), 8.104(\mathrm{~d}, 1 \mathrm{H}, \mathrm{J}=8 \mathrm{~Hz}), 8.349(\mathrm{~s}, 1 \mathrm{H}) ;{ }^{13} \mathrm{C}-\mathrm{NMR}\left(\mathrm{CDCl}_{3}, 100 \mathrm{MHz}\right): 77.6$, $123.9,124.27,125.7,126.3,127.3,128.5,129.2,130.7,133.24,140,142.4,149.5 ; \mathrm{IR}\left(\mathrm{KBr}, \mathrm{cm}^{-1}\right)(\mathrm{C}-\mathrm{H}) 2975$,

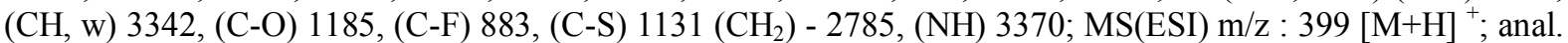
Calculated for $\mathrm{C}_{24} \mathrm{H}_{15} \mathrm{FN}_{2} \mathrm{OS}$; C, 72.34; H, 3.79; F, 4.77; N, 7.03; O, 4.02; S, 8.05; found C, 72.35; H, 3.81; F, $4.79 ; \mathrm{N}, 7.05 ; \mathrm{O}, 4.05 ; \mathrm{S}, 8.07$.

\subsubsection{2-Biphenyl-3-yl-5-(5-phenyl-thiophen-2-yl)-[1,3,4]oxadiazole(7c):}

Pale yellow solid; Yield 66\%; m. p 132-133 ${ }^{0} \mathrm{C} ;{ }^{1} \mathrm{H}-\mathrm{NMR}\left(400 \mathrm{MHz}, \mathrm{CDCl}_{3}\right): \delta 7.386(\mathrm{t}, 2 \mathrm{H}, \mathrm{J}=8.4 \mathrm{~Hz}), 7.451$ $(\mathrm{m}, 3 \mathrm{H}, \mathrm{J}=26.4 \mathrm{~Hz}), 7.613(\mathrm{t}, 1 \mathrm{H}, \mathrm{J}=15.6, \operatorname{Ar}-\mathrm{H}), 7.688(\mathrm{t}, 4 \mathrm{H}, \mathrm{J}=8.4 \mathrm{~Hz}), 7.782\left(\mathrm{td}, 1 \mathrm{H}, \mathrm{J}=10.4 \mathrm{~Hz}, \mathrm{~J}^{\prime}=\right.$ $2.8 \mathrm{~Hz}), 7.825\left(\mathrm{dd}, 1 \mathrm{H}, \mathrm{J}=4 \mathrm{~Hz}, \mathrm{~J}^{\prime}=1.6 \mathrm{~Hz}\right), 8.351(\mathrm{t}, 1 \mathrm{H}, \mathrm{J}=3.2 \mathrm{~Hz}) ;{ }^{13} \mathrm{C}-\mathrm{NMR}\left(\mathrm{CDCl}_{3}, 100 \mathrm{MHz}\right): 77.15,123.9$, $124.23,125.75,126.29,127.36,128.5,129.40,130.72,133.24,140.0,142.4,160.9,164.12 ; \mathrm{IR}\left(\mathrm{KBr}, \mathrm{cm}^{-1}\right)(\mathrm{C}-$ H) 2985, (CH, w) 3352, (C-O) 1195, (C-F) 887, (C-S) $1136\left(\mathrm{CH}_{2}\right)$ - 2787, (NH) 3374; MS(ESI) m/z : 381 $[\mathrm{M}+\mathrm{H}]^{+}$; anal. Calculated for $\mathrm{C}_{24} \mathrm{H}_{16} \mathrm{~N}_{2} \mathrm{OS} ; \mathrm{C}, 75.77 ; \mathrm{H}, 4.24 ; \mathrm{N}, 7.36 ; \mathrm{O}, 4.21 ; \mathrm{S}, 8.43$; found $\mathrm{C}, 75.78 ; \mathrm{H}$, $4.25 ; \mathrm{N}, 7.38 ; \mathrm{O}, 4.24 ; \mathrm{S}, 8.44$.

\subsubsection{2-(4'-Fluoro-biphenyl-2-yl)-5-(5-phenyl-thiophen-2-yl)-[1,3,4]oxadiazole(7d):}

Yellow solid; Yield 66\%; m. p 132-133 ${ }^{0} \mathrm{C}$; ${ }^{\mathrm{H}} \mathrm{H}-\mathrm{NMR}\left(400 \mathrm{MHz}, \mathrm{CDCl}_{3}\right): \delta 7.080(\mathrm{t}, 2 \mathrm{H}, \mathrm{J}=17.6 \mathrm{~Hz}), 7.249$ (q, $5 \mathrm{H}), 7.326(\mathrm{~d}, 1 \mathrm{H}, \mathrm{J}=7.2 \mathrm{~Hz}, \operatorname{Ar}-\mathrm{H}), 7.392(\mathrm{q}, 3 \mathrm{H}), 7.497(\mathrm{t}, 1 \mathrm{H}, \mathrm{J}=14.4 \mathrm{~Hz}), 7.565(\mathrm{~m}, 3 \mathrm{H}, \mathrm{J}=12.4 \mathrm{~Hz}), 7.779$ $(\mathrm{d}, 1 \mathrm{H}, \mathrm{J}=8 \mathrm{~Hz}), 8.125(\mathrm{~d}, 1 \mathrm{H}, \mathrm{J}=7.2 \mathrm{~Hz}) ;{ }^{13} \mathrm{C}-\mathrm{NMR}\left(\mathrm{CDCl}_{3}, 100 \mathrm{MHz}\right): 21.6,77.15,115.3,122.6,123.5,124.0$, $126.3,128.4,129.5,130.42,131.5,133.1,136.7,137.5,141.0,143,149.4,161.3,163.8,182.9 ; \mathrm{IR}\left(\mathrm{KBr}, \mathrm{cm}^{-1}\right)$ (C-H) 2965, (CH, w) 3372, (C-O) 1198, (C-F) 889, (C-S) $1146\left(\mathrm{CH}_{2}\right)$ - 2797, (NH) 3394; MS (ESI) m/z : 399 $[\mathrm{M}+\mathrm{H}]{ }^{+}$; anal. Calculated for $\mathrm{C}_{24} \mathrm{H}_{15} \mathrm{FN}_{2} \mathrm{OS} ; \mathrm{C}, 72.34 ; \mathrm{H}, 3.79 ; \mathrm{F}, 4.77 ; \mathrm{N}, 7.03 ; \mathrm{O}, 4.02 ; \mathrm{S}, 8.05$; found $\mathrm{C}$, 72.36; H, 3.80; F, 4.78; N, 7.06; O, 4.04; S, 8.06.

\subsubsection{2-(4-Methoxy-phenyl)-5-(5-phenyl-thiophen-2-yl)-[1,3,4]oxadiazole(7e):}

Yellow solid; Yield 56\%; m. p 142-148 ${ }^{0} \mathrm{C}$; ${ }^{1} \mathrm{H}-\mathrm{NMR}\left(400 \mathrm{MHz}, \mathrm{CDCl}_{3}\right): \delta 3.893\left(\mathrm{~s}, 3 \mathrm{H}, \mathrm{OCH}_{3}\right), 7.030(\mathrm{~d}, 2 \mathrm{H}, \mathrm{J}$ $=9.2 \mathrm{~Hz}), 7.303(\mathrm{~d}, 2 \mathrm{H}, \mathrm{J}=8.4 \mathrm{~Hz}, \mathrm{Ar}-\mathrm{H}), 7.37(\mathrm{~d}, 1 \mathrm{H}, \mathrm{J}=4 \mathrm{~Hz}), 7.430$ (q, 2H, J = $14.8 \mathrm{~Hz}), 7.662(\mathrm{~d}, 2 \mathrm{H}, \mathrm{J}=$ $7.2 \mathrm{~Hz}), 7.77(\mathrm{~d}, 1 \mathrm{H}, \mathrm{J}=3.6 \mathrm{~Hz}), 7.811(\mathrm{~d}, 2 \mathrm{H}, \mathrm{J}=8.4 \mathrm{~Hz}), 8.059(\mathrm{~d}, 2 \mathrm{H}, \mathrm{J}=8.8 \mathrm{~Hz}) ;{ }^{13} \mathrm{C}-\mathrm{NMR}\left(\mathrm{CDCl}_{3}, 100 \mathrm{MHz}\right)$ : 21.6, 55.6, 77.15, 114.6, 116.2, 124.0, 126.39, 128.8, 129.5, 130.5, 133.2, 139.2, 143.6, 149.1, 162.5; IR (KBr, $\mathrm{cm}^{-1}$ ) (C-H) 2965, (CH, w) 3372, (C-O) 1198, (C-F) 889, (C-S) $1146\left(\mathrm{CH}_{2}\right)-2797,(\mathrm{NH}) 3394 ; \mathrm{MS}(\mathrm{ESI}) \mathrm{m} / \mathrm{z}$ : $399[\mathrm{M}+\mathrm{H}]^{+}$; anal. Calculated for $\mathrm{C}_{19} \mathrm{H}_{14} \mathrm{~N}_{2} \mathrm{O}_{2} \mathrm{~S} ; \mathrm{C}, 68.24 ; \mathrm{H}, 4.22 ; \mathrm{N}, 8.38 ; \mathrm{O}, 9.57 ; \mathrm{S}, 9.59$; found C, 68.26; H, $4.24 ; \mathrm{N}, 8.39 ; \mathrm{O}, 9.58 ; \mathrm{S}, 9.60$. 
1.4 Scheme 1: Synthesis of novel derivatives of 1,3,4-oxadiazole containing 5-phenylthiophene moiety 7a-e:

(a-e

$+$
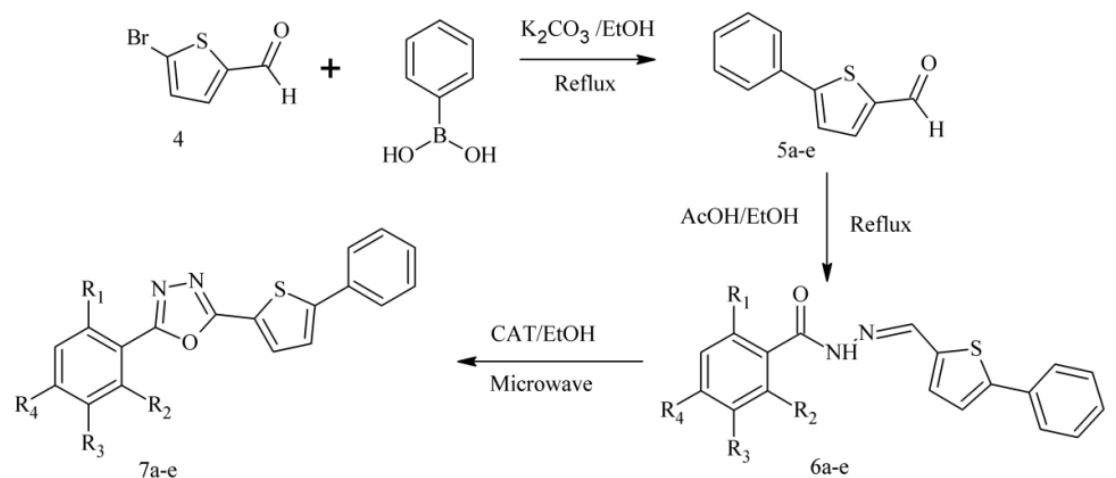

(a): $\mathrm{R}_{1}=\mathrm{OCH}_{3}, \mathrm{R}_{2}=\mathrm{H}, \mathrm{R}_{3}=\mathrm{OCH}_{3}, \mathrm{R}_{4}=\mathrm{H}$

(b): $\mathrm{R}_{1}=\mathrm{H}, \mathrm{R}_{2}=\mathrm{H}, \mathrm{R}_{3}=\mathrm{Ph}-\mathrm{F}, \mathrm{R}_{4}=\mathrm{H}$

(c): $\mathrm{R}_{1}=\mathrm{H}, \mathrm{R}_{2}=\mathrm{H}, \mathrm{R}_{3}=\mathrm{Ph}, \mathrm{R}_{4}=\mathrm{H}$

(d): $\mathrm{R}_{1}=\mathrm{H}, \mathrm{R}_{2}=\mathrm{Ph}-\mathrm{F}, \mathrm{R}_{3}=\mathrm{H}, \mathrm{R}_{4}=\mathrm{H}$

(e): $\mathrm{R}_{1}=\mathrm{H}, \mathrm{R}_{2}=\mathrm{H}, \mathrm{R}_{3}=\mathrm{H}, \mathrm{R}_{4}=\mathrm{OCH}_{3}$

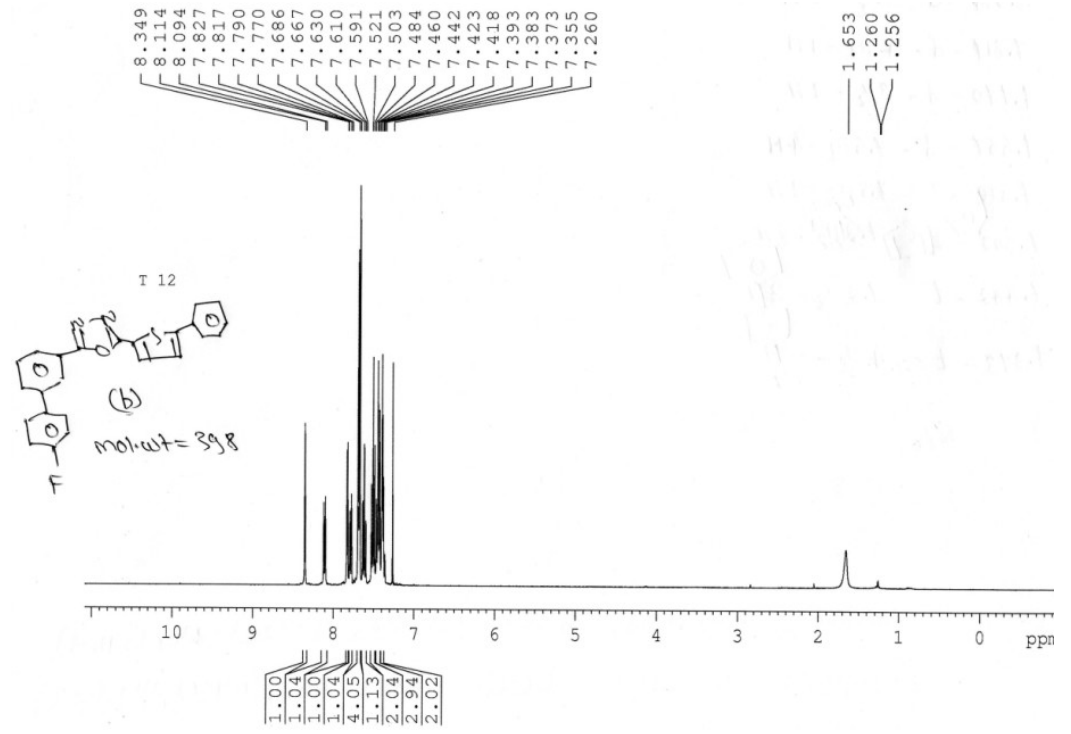



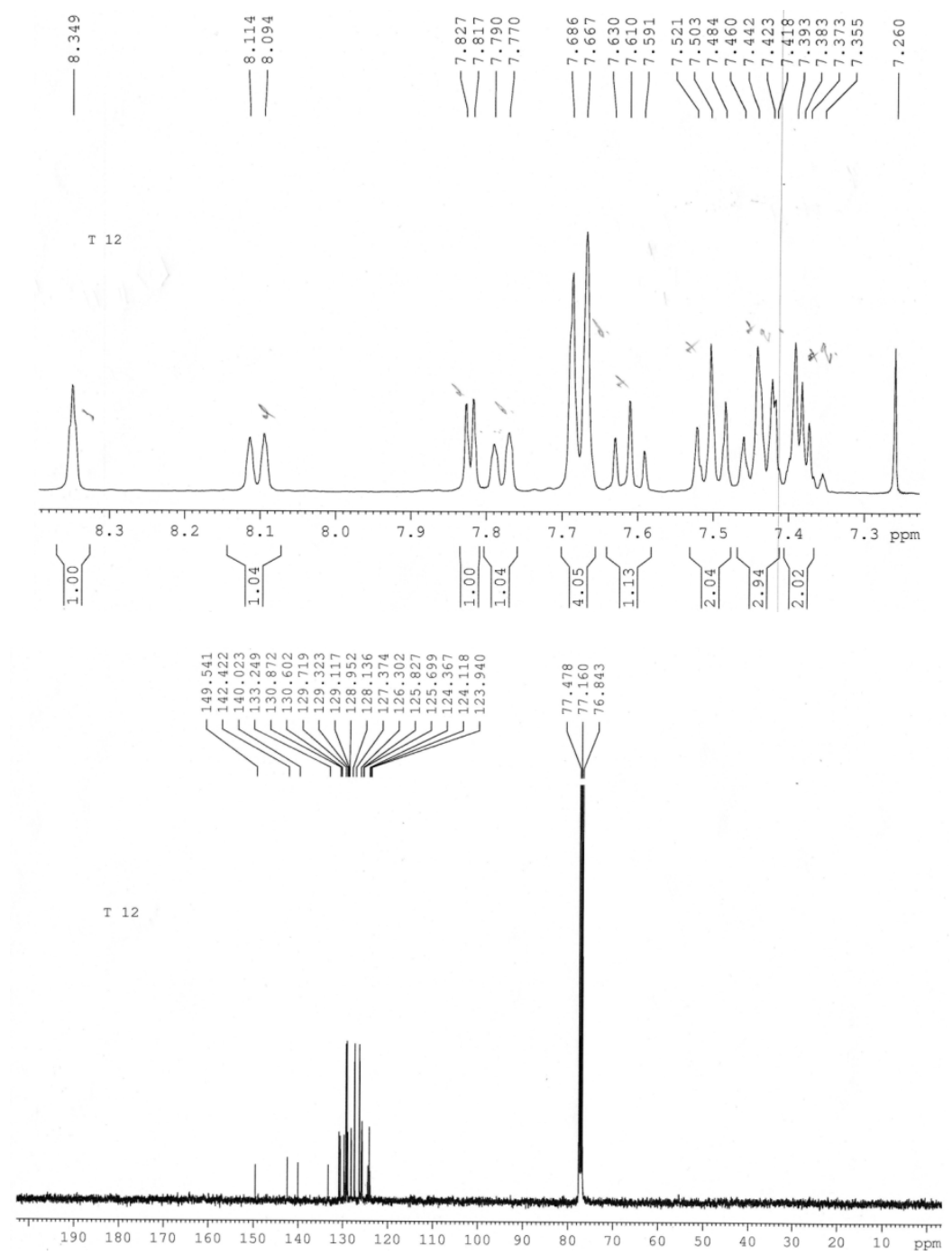

1.4.1 Figure 2: ${ }^{1} \mathrm{H}-\mathrm{NMR}$ and ${ }^{13} \mathrm{C}$ NMR spectra of the most active compound on Caco-2, 7(b).

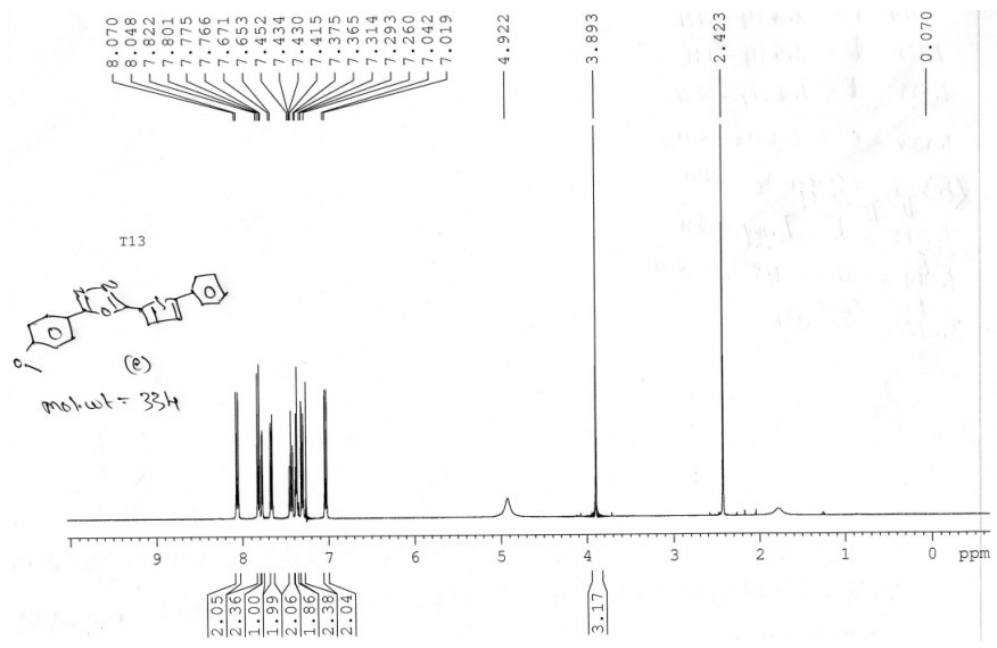




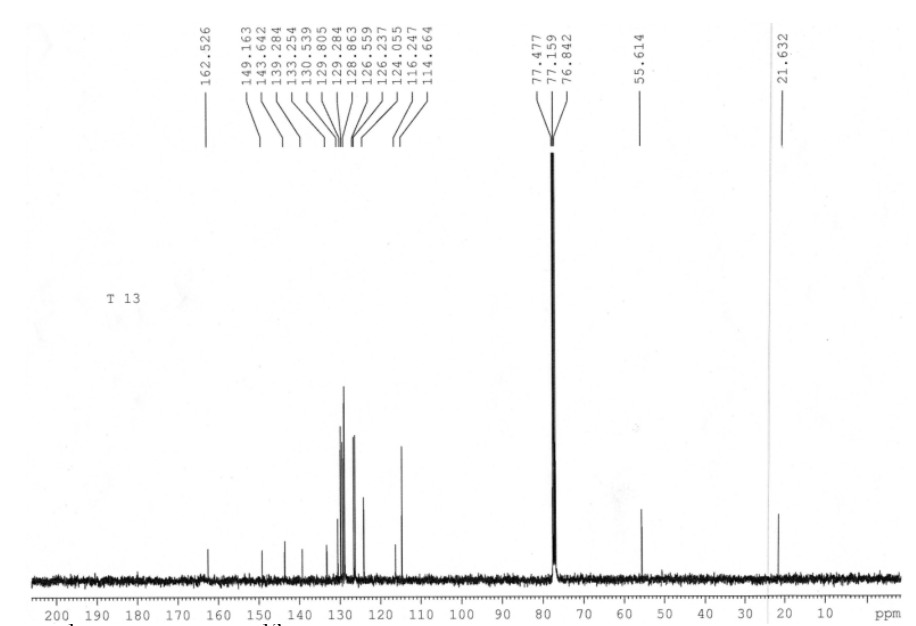

2.4.2 Figure 3: ${ }^{20} \mathrm{H}-\mathrm{NMR}$ and ${ }^{13} \mathrm{CNMR}$ spectra of the moderately active compound 7 (e):

Table 1: $\mathrm{IC}_{50}$ Values of the Novel Derivatives of 1,3,4-Oxadiazole (7a-e) Containing 5-Phenyl Thiophene Moiety:

\begin{tabular}{|l|l|l|l|l|}
\hline \multirow{2}{*}{ SI. No } & \multirow{2}{*}{ Compounds } & \multicolumn{2}{|l|}{ IC50 Values of 1,3,4-Oxadiazoles 7a-e $(\boldsymbol{\mu M})$} \\
\cline { 3 - 5 } & & HepG2 $(\boldsymbol{\mu M})^{*}$ & $\begin{array}{l}\text { Caco-2 } \\
(\boldsymbol{\mu M}) *\end{array}$ & PANC-1( $\boldsymbol{\mu M})^{*}$ \\
\hline 1 & $7 \mathrm{a}$ & 67.8 & 78.9 & 112.3 \\
\hline 2 & $7 \mathrm{~b}$ & 78.9 & $5.3 *$ & 34.5 \\
\hline 3 & $7 \mathrm{c}$ & 125.6 & 56.6 & 56.8 \\
\hline 4 & $7 \mathrm{~d}$ & 234.5 & 78.5 & 123.6 \\
\hline 5 & 7e & 28.4 & 156.7 & 234.6 \\
\hline 6 & 5-fluouracil & $\mathbf{6 . 7}$ & $\mathbf{8 . 6}$ & $\mathbf{7 . 3}$ \\
\hline
\end{tabular}

5-fluorouracil-standard used; *potent molecule; $\mathrm{IC}_{50}$ : Inhibitory concentration of the compound at $50 \%$ of the cells.

\subsubsection{Cell Lines fixation and Culture Conditions:}

\section{Cytotoxic Evaluation (Table 1):}

The in vitro anti-proliferative study was carried out on three human cancer cell lines namely HepG2, Caco-2 and PANC-1. The cell lines were grown in DMEM-HG supplemented with $10 \%$ heat-inactivated FBS, $2 \%$ Penicillin-Streptomycin and $2.5 \mu \mathrm{g} / \mathrm{mL}$ Amphotericin-B solutions (All from HI Media Labs, Mumbai, India). Cell lines were incubated at $37^{\circ} \mathrm{C}$ in a humidified atmosphere of $95 \%$ air, $5 \% \mathrm{CO}_{2}$. Following $24-48 \mathrm{hrs}$ of incubation period, the adherent cells were detached using Trypsin-EDTA solution (HI Media Labs, Mumbai, India). Cell count was done using the Luna automated cell counter (Logos Biosystems, India) based on trypan blue dye exclusion method. Cytotoxicity of the novel 1, 3, 4-oxadiazoles have been determined using MTT 3-(4, 5-Dimethylthiazol-2-yl)-2, 5-diphenyltetrazolium bromide) assay.

3.1.2 Cell Viability Assay (MTT Assay): The MTT assay was carried out at Genelon Institute of Life Sciences Pvt. Ltd. 200 $\mu$ L cell suspension was seeded in 96-well microplates (Corning®, USA) at a density of 25,000 cells/well and incubated for $24 \mathrm{hrs}$, all cells were seeded in duplicate with novel compounds $7 \mathrm{a}-\mathbf{e}$ having range of concentrations from $50 \mu \mathrm{M}-500 \mu \mathrm{M}$, incubated in a $\mathrm{CO}_{2}$ incubator at $37^{\circ} \mathrm{C}$. Treated cells were thereafter incubated with $10 \%$ MTT (5mg/ml; HI Media Labs, Mumbai, India) for 3 hrs. The culture medium was then aspirated and $200 \mu \mathrm{L}$ dimethyl sulfoxide (DMSO; Sigma-Aldrich, India) was added. 5-Fluoro uracil (5-FU) was used as standard. Cell viability was determined by measuring the absorbance on a micro plate reader (SPECTRO STAR NANO, BMG LABTECH, Germany) at $570 \mathrm{~nm}$. Cell viability was calculated using the formula $\left[\%\right.$ cell viability $=\left(\mathrm{A}_{570}\right.$ of treated cells $/ \mathrm{A}_{570}$ of control cells $\left.) \times 100 \%\right]$.

\section{Results And Discussions:}

4.1 Chemistry (Scheme 1):In the present work novel derivatives of 1, 3, 4-oxadiazoles 7a-e were synthesized, characterized and evaluated for their cytotoxicity against HepG2, Caco-2 and PANC-1 cell lines. Synthetic chemistry involved the conversion of substituted acids into corresponding ester 2a-e. The ester compound was further converted into corresponding carbohydrazide (IR absorbance of NH $3345 \mathrm{~cm}^{-1}$ ) 3a-e. The carbohydrazide 3a-e was reacted with 5-phenylthiophene carboxaldehde and obtained Schiff base ${ }^{[17]}$ compounds 6a-e. The Schiff base compounds were cyclized in presence of chloramine-T as promoter and obtained the novel series of 1,3,4-oxadiazoles 7a-e. The synthesized novel derivatives of 1,3,4-oxadiazole compounds were evaluated for their cytotoxicity ${ }^{[17,18]}$ against HepG2, Caco-2 and PANC-1 cell lines using MTT assay. 
4.2 b) Anticancer activity (Table 1): The synthesized compounds were screened for in vitro anticancer activity against three human cancer cell lines ${ }^{[18,19]}$ namely, HepG2, Caco-2 and PANC-1. The results were expressed in the form of $\mathrm{IC}_{50}$ (Inhibitory concentration of the compound at 50\%). The 5-fluorouracill was used as a standard. The compound (7b) (Fig. 2)showed cytotoxicityagainst human colorectal carcinoma cell line (Caco-2) with $\mathrm{IC}_{50}$ of $5.3 \mu \mathrm{M}$ which is comparable to that of know standard 5-fluorouracil. The compound 7(e) (Fig. 3) showed moderatecytotoxicity ${ }^{[20]}$ against $\mathrm{HepG} 2$ cell line with $\mathrm{IC}_{50}$ of $28.4 \mu \mathrm{M}$.

\section{Conclusion:}

The cytotoxicity of the synthesized 1,3,4-oxadiazole compounds 7a-e were compared with the cytotoxicity of the known standard, 5-fluorouracil.Cytotoxicity of the compound 7b (Fig. 2)against Caco-2cell line with $\mathrm{IC}_{50}$ of $5.3 \mu \mathrm{M}$ which is comparable with the cytotoxicity of the known standard 5-fluorouracil. The compound 7e (Fig. 3) showed moderate cytotoxicity against $\mathrm{HepG} 2$ cell line whose $\mathrm{IC}_{50}$ value is $28.4 \mu \mathrm{M}$. The authors are interested to study the various biological activities and apoptosis mechanism in their further research.

\section{Acknowledgements}

Author is thankful to Mount Carmel College (Autonomous), Bangalore, India. Authors also thank Mr.Vinod H Naik for the analytical data.

\section{References}

[1]. Nagalakshmi, G. Synthesis, antimicrobial and antiinflammatory activity of 2, 5-disubstituted-1, 3, 4-oxadiazoles,Indian Journal of Pharmaceutical Sciences., 70(1), 2008, 49-55.

[2]. Jignesh, P.R. Tarunkumar, N. Akhaja, D. Jaspara, M. Kruti, N.M. Nilesh, H.P. Synthesis and in vitro antibacterial activity of new oxoethylthio-1,3,4-oxadiazole derivatives.Journal of Saudi Chemical Society., 18(2), 2014, 101-106

[3]. Jnyanaranjan, P. Jagannath, P.V. Chandra, S.P. Jitendriya, M. Synthesis, Characterization, Antibacterial and Analgesic evaluation of some 1, 3, 4-Oxadiazole Derivatives, Der PharmaChemica., 3, 2011, 485-490

[4]. Janin, Y, Janin. Antituberculosis drugs ten years of research. Bioorganic and Medicinal Chemistry. 15, 2007, 2479-2513..

[5]. Aboraia, A.S. Abdel-Rahman, H.M. Mahfouz, N.M. El-Gendy, M.A. Novel5-(2-hydroxyphenyl)-3-substituted-2,3-dihydro-1,3,4oxadiazole-2-thione derivatives promising anticancer agents. Bio org. Med. Chem.,14(4), 2006, 1236-1246.

[6]. Adimule, V, Medapa, S. Rao, P. K. Kumar, L. S. Synthesis of Schiff bases of 5-[5-(4-fluorophenyl) thiophen-2-yl]-1, 3, 4thiadiazol-2-amine and its anticancer activity. International Journal of Advanced Pharmaceutical Research, 5(1), 2014, $1761-1768$.

[7]. Demirayak, S.Kayagil, I.Yurttas, L., Microwave supported synthesis of some novel 1,3-diarylpyrazino[1,2-a] benzimidazole derivatives and investigation of their anticancer activities,Eur J Med Chem 46(1), 2011, 411-416

[8]. Mamdouh, A.Z. Abu-Zaied, G. A. M, Nawwar. R. H, Swellem. S.H. El-Sayed,Synthesis and Screening of New 5-Substituted-1,3,4oxadiazole-2-thioglycosides as Potent Anticancer Agents Pharmacology \& Pharmacy, 3, 2012, 254-261.

[9]. Mogilaiah, K. Srinivas, R.C. Chloramine-T mediated synthesis of 1, 3, 4-oxadiazolyl-1, 8-naphthyridines under microwave irradiation. Indian Journal of Chemistry, 44(B), 2005, 768-772

[10]. Anees, P.Javed, A. S. Various Pharmacological aspects of 2, 5-Disubstituted 1,3,4-OxadiazoleDerivatives, A Review Research Journal of Chemical Sciences, 3(12), 2013,79-89.

[11]. G. Patrick, S.,Nuleophilicfluoro alkylation of aldehydes, US175302A, 1987July 3.

[12]. Palizban, A.A. Sadeghi-aliabadi, H. Abdollahpour, F. Effect of cerium lanthanide on Hela and MCF-7 cancer cell growth in the presence of transferring. Research Pharma Science.,5(2), 2010, 119-125.

[13]. Irfan A.M. Chloramine-T mediated synthesis of 1, 3, 4-oxadiazoleas antibacterial agents,Der Pharmacia Sinica., 2(6), 2011, 102106.

[14]. Anand, P.Patil, V.M. Sharma, V.K.Khosa, V.K.Masand, N. Schiff bases a review on biological insights,International Journal of Drug Design and Discovery, 3(3), 2012, 851-868.

[15]. Galligan, P. R. Paul, J. McGuirk, M. J. Witty.Process for the preparation of 3-(2'-fluorophenyl) pyridine, US 4797490 A15, 1986

[16]. Shadia, A. Galal, A.S. Abdelsamie, M. L. Rodriguez, S. M. Kerwin and Hoda, I. E. Synthesis and studying the antitumor activity of novel5-(2-methylbenzimidazol-5-yl)-1,3,4-oxadiazole-2(3H)-thiones,European Journal of Chemistry, 1 (2), 2010, 67-72

[17]. Anna, C. Anne, S.V. C. Jean-Charles, L. Maria, S. S. Antonio, G. and Sylvain, R. Efficient and Simple Synthesis of6-Aryl-1,4dimethyl-9H-carbazoles, Molecules,13,2008, 1312-1320.

[18]. Desai, N. C. Bhatt, N.Hardik,S.Amit, T. Synthesis, antimicrobial and cytotoxic activities of some novel thiazole clubbed 1,3,4oxadiazoles. European Journal of Medicinal Chemistry, 67,2013, 54-59.

[19]. Juan, S.Hui Z, Zhong-Ming, Y and Hai-Liang, Z Synthesis, molecular modeling and biological evaluation of 2-aminomethyl-5(quinolin-2-yl)-1,3,4-oxadiazole-2(3H)-thione quinolone derivatives as novel anticancer agent. European Journal of Medicinal Chemistry,60,2013, 23-28.

[20]. Rajesh, A. R.Pavankumar, B.Sheetal, D .B and Preeti, K .K. Synthesis and evaluation of novel 4-nitropyrrole-based 1,3,4oxadiazole derivatives as antimicrobial and anti-tubercular agents. European Journal of Medicinal Chemistry,70,2013, 49-58. 